\title{
Fecal microbiota transplantation: the european consensus and first ukrainian experience
}

\section{Summary}

Transplantation of fecal microbiota: European consensus and first Ukrainian experience Objective: to establish the efficiency of transplantation of fecal microbiota (FMT) in patients with various intestinal diseases.

\begin{abstract}
Material and methods: from the end of 2015 to this time, 46 single procedures of FMT were conducted in one center, of which 22 were fresh material, 24 were frozen material from healthy donors. Selection and preparation of donors for FMT was conducted in accordance with the European consensus on FMT, published in the form of clinical recommendations for physicians in 2017. Among the selected patients were 22 women and 24 men aged 28 to 52years (mean age $-40.2+10.6 y e a r s)$. The main indications for FMT were postinfectious diarrhea-predominent irritable bowel syndrome (IBS-D) - 15 patients, nonspecific ulcerative colitis (UC) - 12 patients, Crohn's disease (CD) - 6 patients, antibiotic-associated diarrhea (AAD) - 5 patients, C. difficile-associated pseudomembranous colitis (PMC) - 4 patients.

Results: in all patients with C.difficile-associated PMC after single FMT, clinical improvement and an outbreak of deep remission that was prolonged at this time was noted. High efficacy of FMT was also noted in AAD (clinical improvement in $80 \%$ of patients), post-infective IBS-D and UC (clinical improvement in $66.6 \%$ of patients). In patients with $\mathrm{CD}$, the efficacy of FMT was less $(50 \%)$, with the immediate side effects in 1 patient were observed in the form of fever and abdominal pain that resolved within 1week. No other significant clinically significant side effects of FMT have been observed in our observations.

Conclusions: taking into account the own experience and recommendations of the European consensus on FMT, it can be concluded that FMT is an important and highly effective therapeutic intervention in the treatment of Clostridium difficile and associated PMC. In addition, FMT is a promising and effective treatment for other disorders associated with changes in intestinal microbiota, in particular inflammatory bowel disease, IBD-D, and $\mathrm{AAD}$.
\end{abstract}

Keywords: fecal microbiota transplantation, intestinal diseases
Volume 10 Issue 3 - 2019

\author{
SMTkach,' YH Kuzenko,' AE Dorofeyev, ${ }^{2} Y Z$ \\ Dynia, ${ }^{2}$ AA Dorofeyeva ${ }^{3}$ \\ 'The Applied Research Center for Endocrine Surgery, Endocrine \\ Organ and Tissue Transplantation of the Ministry of Health of \\ Ukraine, Ukraine \\ ${ }^{2}$ Shupyk National Medical Academy of Postgraduate Education, \\ Ukraine \\ ${ }^{3}$ Institute of Gerontology n.a. D.F.Chebotarev, Ukraine
}

Correspondence: AE Dorofeyev, National Medical Academy of Postgraduate Education n.a.P.L.Shupik, Kiev, Ukraine, Email dorofeyevand@gmail.com

Received: May 03, 2019 | Published: June 03, 2019
Abbreviations: FM, fecal microbiota; FMT, fecal microbiota transplantation; UEG, united european gastroenterology; IBD, inflammatory bowel disease; RCTs, randomized clinical trials; DM, diabetes mellitus; IBS, irritable bowel syndrome

\section{Introduction}

Understanding the role of fecal microbiota (FM) in the pathogenesis of various diseases, in particular gastroenterological and metabolic, as well as the new opportunities for the therapy utilizing FM modification, turned out to be the most crucial among medical breakthroughs over the past decade. ${ }^{1-3}$ The number of clinical trials and publications related is tallied in hundreds of authoritative articles over the past decade. The interest in FM has increased markedly since the first reports on the striking therapeutic efficiency of fecal microbiota transplantation (FMT) in pseudomembranous colitis, as well as other enteropathies refractory to conventional therapy were released. ${ }^{4,5}$

FMT is a single-step therapeutic procedure which enables to restore quickly the normal colon microbiome. In October 2013, at the XXI United European Gastroenterology (UEG) Week FMT was introduced as one of the 3 most significant achievements of gastroenterology in 2013. For several decades FMT has been performed only in few centers worldwide, and generally only as a last resort in patients with relapsing $C$. difficile infection. ${ }^{1,6}$
Currently there are already several hundred publications reporting on both clinical cases and randomized clinical trials (RCTs) that have revealed about $90 \%$ cumulative toxicity of FMT in the treatment of relapsing $C$. difficile infection excluding the clinically relevant adverse reactions. Furthermore, over the last years thousands of successful FMT procedures have been performed worldwide proving to be effective not only in severe relapsing $C$. difficile infection, but also in inflammatory bowel disease (IBD), intestinal dysbiosis and irritable bowel syndrome (IBS). ${ }^{7-9}$ Moreover, the papers appeared demonstrating the FMT efficiency either in non-gastrointestinal diseases - diabetes mellitus (DM) and insulin resistance, obesity, multiple sclerosis, idiopathic thrombocytopenic purpura, etc. ${ }^{1,10}$

Over the last 3years, FMT has been performed systematically in Ukraine. The first-ever Ukrainian experience and FMT outcomes obtained in one center in various pathologies.

The aim of the study was to evaluate the effectiveness and safety of using TM in patients of various diseases, in whom the standard therapy was ineffective.

\section{Materials and methods}

From the end of 2015 until the present day at the Ukrainian Applied Research Center for Endocrine Surgery, Endocrine Organ and Tissue Transplantation of the Ministry of Health of Ukraine 46 single-step FMT procedures were performed, 22 of which using fresh material 
and 24 - frozen fecal samples from healthy donors. There were 22 females and 24 males aged 28-52years (mean age $-40.2 \pm 10.6$ years) among the selected subjects.

FMT was approved by Local Bioethical Committee of Ukrainian Applied Research Center for Endocrine Surgery, Endocrine Organ and Tissue Transplantation of the Ministry of Health of Ukraine (protocol № 4/2015, 08.04.2015) All patients signed informed consent.

FMT therapy was recommended to patients who did not have a clinical response to standard, basic therapy of the disease. In case of IBD (UC and CD) patients did not respond to treatment with mesalazine, topical and systemic steroids. Patients with pseudomembranous colitis and antibiotic-associated diarrhea have not response to vancomycin treatment. Patients with post-infectious IBS-D did not responed to treatment by standard treatment with rifaximine, probiotics, spasmolitics. Patients with diabetes and obesity, had DM type 2 at the stage of subcompensation, but could not achieve weight loss.

Given that all patients selected for FMT had standard therapy that was not effective, comparison with the control group was not used.

Clinical features of the FMT-exposed patients are presented in Table 1. Donor selection and preparation for FMT were carried out in compliance with the European Consensus on the FMT guiding published as clinical guidelines for physicians in 2017. ${ }^{1}$ According to this Consensus the critical issue concerning FMT is the donor selection. Considering that the primary objective is to reduce and prevent adverse effects associated with the administration of the fecal transplant, the staged FMT donor selection was attempted regardless of indications. In order to exclude potential risk factors (Table 2) the survey focused on the potential donor's habits and past medical history was conducted for the reasons of considering the general exclusion criteria for donors prior to the FMT procedure at the first stage. It is critical in order to avoid threats undetectable when analyzing blood and feces. The subjects under 60 were preferable in the attempt to reduce the probability of comorbidities. The monitored donors were additionally interviewed on the date of the test delivery in order to exclude recent and potential life-threatening events. The alternative technique without using a questionnaire was applied when frozen material was used, and involved the sample collection right after obtaining the study results, their subsequent freezing at $-80^{\circ} \mathrm{C}$ and storing for the following use (not more than 2months).

Another critical issue was the donor testing. In compliance with the guidelines, FMT-eligible donors delivered their blood and stool samples not later than 4weeks prior to the date of the delivery (Table 3 ). If the changes in donor's overall health have not been reported, the tests were repeated in 8 weeks. Preparation of the stool sample is the further critical issue when performing FMT. Although the protocols used in various studies are similar, we stuck to the certain phasing, presented in Table 4, for fresh stool samples preparation. We used fecal material and sterile $0.9 \%$ saline, in the ratio of 1:5 (30-50g of feces diluted in $150 \mathrm{ml}$ of saline). Following the homogenization the solids were extracted using gauze, tea filter or identical devices, and suspension was transferred to a sterile container.

Glycerol was added to fresh feces before freezing for making up to $10 \%$ in cases of using frozen stool samples in FMT. The resulting suspension was marked (using codes identical to those in blood and organ transplantation) and stored at $-80^{\circ} \mathrm{C}$. On the FMT date, the fecal suspension was thawed in a warm $\left(37^{\circ} \mathrm{C}\right)$ water-bath. Following the thawing a saline was added to make the suspension up to the targeted volume, and thawed fecal material was administered within 6 hours.

Table I Clinical features of the FMT-exposed patients

\begin{tabular}{|c|c|c|c|}
\hline Parameters & & Value & $\begin{array}{l}\% \text { of the } \\
\text { total weight }\end{array}$ \\
\hline Gender (F/M) & & $22 / 24$ & $47.8 / 52.2$ \\
\hline Age, years & & \multicolumn{2}{|c|}{$40.2 \pm 10.6$} \\
\hline \multirow[t]{6}{*}{ Diagnosis } & Ulcerative colitis (UC) & 12 & 26.1 \\
\hline & Crohn's disease (CD) & 6 & 13.04 \\
\hline & Pseudomembranous colitis & 4 & 8.7 \\
\hline & $\begin{array}{l}\text { Antibiotic-associated diarrhea } \\
\text { (AAD) }\end{array}$ & 5 & 10.9 \\
\hline & $\begin{array}{l}\text { Post-infectious IBS } \\
\text { accompanied by diarrhea }\end{array}$ & 15 & 32.6 \\
\hline & $\begin{array}{l}\text { Metabolic disorders (type } 2 \\
\text { DM + obesity) }\end{array}$ & 4 & 8.7 \\
\hline $\begin{array}{l}\text { FMT - } \\
\text { fresh samples }\end{array}$ & & 22 & 47.8 \\
\hline $\begin{array}{l}\text { FMT - } \\
\text { frozen samples }\end{array}$ & & 24 & 52.2 \\
\hline
\end{tabular}

Table 2 Principal selective restrictions concerning FMT donors Infectious reasons

\section{Infectious reasons}

Current diseases from the past medical history (HIV, HBV or HCV, syphilis, type I and 2 human T-lymphotropic virus, malaria, trypanosomiasis, tuberculosis)

Diagnosed uncontrolled infectious disease

Drug addiction

Sexual behavior associated with a high risk of sexually transmitted diseases History of organ/tissue transplantation

Blood transfusion ( $<12$ months)

Accidental needlestick injury (<6months)

Tattoo, piercing, etc. (<6months)

Prion disease risk

Previous parasitic disease, retrovirus infection, Giardia lamblia and other infectious and parasitic diseases involving gastrointestinal tract

Recent (<6months) tropical trips to high infectious risk countries or traveler's diarrhea

Previous (<6months) vaccination with live attenuated virus

Health professionals (in order to eliminate the risk of transmitting the multidrug-resistant organisms)

Individual work with animals (in order to eliminate the risk of zoonotic diseases)

\section{Gastrointestinal, metabolic or neurological diseases}

IBD, IBS, chronic functional constipation, celiac disease, and history of other gastrointestinal diseases 


\section{Infectious reasons}

History of chronic systemic autoimmune diseases involving gastrointestinal tract

High risk of gastrointestinal cancer and polyposis

Recent diarrhea or hematochezia

History of neural, neurodegenerative and mental disorders

Overweight and obesity (body mass index $>25$ )

Use of the drugs that impact the intestinal microbiome composition

Recent (<3months) use of antibiotics or immunosuppressants, chemotherapy

Long-term PPI use

Table 3 Blood and stool tests delivered in donors for any potentially transmitted diseases

\section{Blood test \\ Complete blood count \\ Cytomegalovirus (CMV) \\ Epstein-Barr virus (EBV) \\ Hepatitis A \\ HBV, HCV, HEV \\ Syphilis \\ HIV-I and HIV-2 \\ Entamoeba histolytica \\ C-reactive protein (CRP) \\ Liver function tests, albumin \\ Creatinine and electrolytes}

\section{Stool tests}

Stool tests for Clostridium difficile toxin, enteric pathogens including Salmonella, Shigella, Campylobacter, Escherichia coli OI 57 H7, Yersinia, vancomycin-resistant Enterococcus, methicillin-resistant Staphylococcus aureus, multidrug-resistant Gram-negative bacteria, Norovirus

Giardia lamblia and Cryptosporidium parvum antigens

Protozoa (including Blastocystis hominis) and parasitic worms

Fecal occult blood test

Prepared $150 \mathrm{ml}$ of the solution was administered to the patient in the dome of the cecum during colonoscopy. Preparation for colonoscopy was performed using a standard macrogol 4000 solution. During the colonoscopy, the patient was in a state of propofol sedation.

The FMT efficiency was being assessed over the period of 1 month by clinical features (clinical response or its absence, remission), safety and tolerability - by the adverse effects. At this time, the patients did not receive any additional drug therapy. Repeated microbiological examination of the patients was not conducted, except for patients with pseudomembranous colitis induced by $C$. difficile infection.
Table 4 Mandatory steps for preparation of fresh and frozen fecal materials

\section{Fresh feces}

Fresh feces should be utilized within 6 hours after defecation

Preparation and preservation should be carried out as quickly as possible in order to protect anaerobic bacteria

Stool sample can be stored at ambient temperature of $20^{\circ} \mathrm{C}-30^{\circ} \mathrm{C}$ prior to the subsequent processing

Minimum amount $(30 \mathrm{~g})$ of feces is commonly utilized

Fecal material should be whipped in a saline using a blender and followed by sieving to avoid infusion syringe and catheter clogging

Sterile room subjecting to the effective measures against sporeforming bacteria should be used

Protective gloves and face mask should be worn during the preparation

\section{Frozen fecal material}

At least $30 \mathrm{~g}$ of donor feces and $150 \mathrm{ml}$ of saline should be utilized

It is required to make glycerol up to $10 \%$ of the final solution prior to freezing

Resulting suspension should be strictly marked and stored at $80^{\circ} \mathrm{C}$

Fecal suspension should be thawed in a warm $\left(37^{\circ} \mathrm{C}\right)$ water-bath and administered within 6 hours after the thawing on the date of FMT

Saline is commonly added to make the suspension up to the targeted volume after the thawing

Avoid freeze-thaw cycles

\section{Results}

FMT results in various pathological conditions are presented in Table 5. The above results suggest that the clinical response and long-lasting remission were reported in all patients with $C$. difficile induced pseudomembranous colitis after single-step FMT, moreover, the relapse has not been reported so far. In all these patients microbiological test for C.difficile after 1 month were negative. The high FMT efficiency also took place in AAD (clinical response in $80 \%$ of patients). In one patient with antibiotic-associated diarrhea, despite some clinical improvement, recurrent episodes of diarrhea were noted. After 7months, this patient had abdominal pain, which was associated with impaired stool frequency and stool consistency. The patient was diagnosed with post-infectious IBS-D.

Among 15patients with post-infectious IBS, the effectiveness of FMT was noted in $10(66.7 \%)$. These patients had no stool abnormalities or episodes of abdominal pain for 6 months. At the same time, despite some clinical improvement, a decrease in the intensity of abdominal pain, a tendency to normalize the consistency of feces, $5(33.3 \%)$ patients with post-infectious IBS had episodes of diarrhea with abdominal pain in the first month. Among 12 patients with UC, in whom therapy with mesalazine in combination with budesonide or systemic steroids was not effective, $8(66.7 \%)$ patients had clinical 
remission after FMT. It should be noted that patients with UC had a moderate course of the disease with a left-sided lesion of the large intestine. During the first month after FMT, patients reported normalization of stool frequency and the absence of blood admixture in feces. The duration of clinical remission was observed in patients with UC from 4months to a year. In addition, in patients with UC, in whom FMT proved to be effective, sensitivity to basic therapy drugs increased. After the onset of exacerbation of UC, it was sorely stopped by the use of standard therapy, which was previously not effective. In patients with Crohn's disease the FMT efficiency was lower (50\%), moreover, the early adverse effects such as fever and increasing abdominal pain were reported in 1 patient within 3days following the intervention, which then resolved over the period of 1week. Any other clinically meaningful FMT adverse effects have not been taken place during the follow-up.

Table 5 FMT efficiency in various pathological conditions

\begin{tabular}{lll}
\hline Diagnosis & $\begin{array}{l}\text { Number of } \\
\text { patients }\end{array}$ & $\begin{array}{l}\text { Clinical response/ } \\
\text { remission }\end{array}$ \\
\hline Ulcerative colitis (UC) & 12 & $8(66.7 \%)$ \\
Crohn's disease & 6 & $3(50 \%)$ \\
$\begin{array}{l}\text { Pseudomembranous colitis } \\
\begin{array}{l}\text { Antibiotic-associated diarrhea } \\
\text { (AAD) }\end{array}\end{array}$ & 4 & $4(100 \%)$ \\
$\begin{array}{l}\text { Post-infectious IBS } \\
\text { accompanied by diarrhea }\end{array}$ & 15 & $4(80 \%)$ \\
$\begin{array}{l}\text { Metabolic disorders } \\
\text { (type 2 DM + obesity) }\end{array}$ & 4 & $10(66.7 \%)$ \\
\hline
\end{tabular}

For the objectification of the obtained results in patients with IBD pseudomembranous colitis, antibiotic-associated diarrhea and postinfectious IBS with diarrhea routine tests and analysis before and after FMT were performed. Stool frequency and presence blood, mucus in stool, feces consistency due to Bristol scale were analyzed. Intensity of abdominal pain syndrome due to visual analog scale (VAS) was determined. Fecal calprotectine levels in stool before and 1 month after FMT were studied (Table 6). After FMT, the clinical condition of the patients improved, but a month after the procedure, only some patients maintained clinical remission.

In UC patients 1month after FMT stool frequency was significantly less, than before treatment. At the same time in $4(33.3 \%)$ patients traces of blood in stool were found, it was a reason for administration anti-inflammatory treatment for these patients. However, fecal calprotectine level after FMT was significantly lower in all UC patients $(98.9 \pm 9.7 \mathrm{mkg} / \mathrm{g}$ and $452.2 \pm 47.7 \mathrm{mkg} / \mathrm{g}$ respectively $(\mathrm{p}<0.05)$. In Crohn's disease patients after FMT intensity of abdominal pain syndrome and fecal calprotectine level were significantly lower than before treatment.

In all patients with $C$. difficile induced pseudomembranous colitis after FMT stable clinical remission with normalization of stool frequency, feces consistency and absents pathological traces in stool were observed. Abdominal pain syndrome in these patients practically was absent, but in 1 patient abdominal discomfort was observed. Level of fecal calprotectine in this group of patients after FMT normalized. In patients with AAD similar clinical findings after FMT were observed. Stool frequency and feces consistency with facal calprotectine level were normalized; pathological traces in stool were absent. However, in 1 patient with AAD abdominal pain syndrome still present what was combined with episodes of diarrhea.

In patients with post-infectious IBS with diarrhea after FMT significant reduction of abdominal pain syndrome intensity with fecal calprotectine normalization were observed. Tendencies to decreasing stool frequency and feces consistency were found in this group of patients. In post-infectious IBS patients after FMT mucus traces in stool was not observed.

A small number of patients (4 subjects) and relatively short-term follow-up are still insufficient to draw reliable conclusions regarding the FMT efficiency in metabolic disorders.

Table 6 Clinical changes in patients before and after FMT

\begin{tabular}{|c|c|c|c|c|c|c|c|c|c|c|}
\hline & \multicolumn{2}{|c|}{$\begin{array}{l}\text { Ulcerative colitis ( } \\
n-12 \text { ) }\end{array}$} & \multicolumn{2}{|c|}{ Crohn's disease $(n-6)$} & \multicolumn{2}{|c|}{$\begin{array}{l}\text { Pseudomembranous } \\
\text { colitis (n-4) }\end{array}$} & \multicolumn{2}{|c|}{$\begin{array}{l}\text { Antibiotic-associated } \\
\text { diarrhea }(n-5)\end{array}$} & \multicolumn{2}{|c|}{$\begin{array}{l}\text { Post-infectious IBS } \\
\text { with diarrhea (n-15) }\end{array}$} \\
\hline & before & after & before & after & before & after & before & after & before & after \\
\hline Stool frequency & $6.2 \pm 0.7$ & $3.6 \pm 0.7 *$ & $5.5 \pm 0.6$ & $2.9 \pm 0.3$ & $6.7 \pm 0.9$ & $1.1 \pm 0.2 *$ & $5.6 \pm 0.7$ & $1.9 \pm 0.2 *$ & $5.6 \pm 0.7$ & $2.7 \pm 0.3$ \\
\hline $\begin{array}{l}\text { Blood, mucus in } \\
\text { stool }\end{array}$ & $4.2 \pm 0.5$ & $1.4+0.1^{*}$ & $2.7 \pm 0.3$ & $1.3 \pm 0.2$ & $3.1 \pm 0.4$ & $-*$ & $2.3 \pm 0.3$ & - & $1.2 \pm 0.2$ & - \\
\hline $\begin{array}{l}\text { Feces } \\
\text { consistency }\end{array}$ & $6.2 \pm 0.3$ & $3.5 \pm 0.6$ & $5.7 \pm 0.7$ & $4.1 \pm 0.6$ & $6.4 \pm 0.6$ & $3.0 \pm 0.4$ & $5.8 \pm 0.6$ & $3.4 \pm 0.4$ & $5.2 \pm 0.6$ & $2.9 \pm 0.4$ \\
\hline $\begin{array}{l}\text { Intensity of } \\
\text { abdominal pain } \\
\text { (score points) }\end{array}$ & $6.1 \pm 0.6$ & $1.9 \pm 0.2 *$ & $7.7 \pm 0.9$ & $2.2 \pm 0.3^{*}$ & $5.9 \pm 0.7$ & $0.5 \pm 0.1 *$ & $5.0 \pm 0.6$ & $1.2 \pm 0.1$ & $8.1 \pm 0.9$ & $2.5 \pm 0.4^{*}$ \\
\hline $\begin{array}{l}\text { Fecal } \\
\text { calprotectine } \\
\text { (mkg/g) }\end{array}$ & $452.2 \pm 47.7$ & $98.9 \pm 9.7^{*}$ & $138.7 \pm 15.1$ & $58.2 \pm 6.9 *$ & $89.9 \pm 9.4$ & $23.2 \pm 2.1 *$ & $64.1 \pm 8.2$ & $12.4 \pm 2.2$ & $59.1 \pm 6.1$ & $16.1 \pm 2.0 *$ \\
\hline
\end{tabular}

*-significant differences between group before and after FMT $(p<0.05)$ 


\section{Discussion}

Although the ever-growing use of FMT is limited due to various bureaucratic hurdles (mainly tied to the donor selection and safety management), the foreign FMT practice is thriving. Since this therapeutic intervention had gained unprecedented popularity, it became necessary to design official evidence-based guidelines. The FMT critical issues, in particular indications for its performing, donor selection, fecal material preparation and clinical monitoring were discussed by 28 experts from 10 European countries at the specially convened European conference held at the end of 2017. As a result, the European Consensus on the FMT guiding was adopted and published as clinical guidelines for physicians, and its highlights referring to donors and fecal material preparation were presented above (Tables $1-3){ }^{1}$

Currently the main and only officially approved indication for FMT is still pseudomembranous colitis (PC) caused by $C$. difficile infection. The guidelines emphasize that the transfer of fecal microbiota from a healthy donor to a recipient in FMT, potentially leads to the normal microbiome restoration in the affected colon and tends to reverse symptoms completely. Several open-label RCTs were indicative of a higher FMT-induced recovery in PC comparing to vancomycin ( $94 \%$ and $90 \%$ in contrast to $31 \%$ and $26 \%$ respectively), and therefore these trials were even suspended. ${ }^{11,12}$ In a few systematic reviews and metaanalyses the FMT-induced recovery from PC varied from $85 \%$ to $89.7 \%{ }^{2-4,7}$ FMT demonstrated the excellent safety at least, in the short term, as only several cases took place when the mild adverse effects were reported. ${ }^{10}$ Although theoretically the transmission of potentially dangerous pathogens, which were inactive over the decades, is considered as possible, this risk should be accounted in the context of favorable risk-benefit ratio, as FMT is the most effective therapeutic intervention in PC and life-saving therapy for these patients. From this perspective, the widespread adoption of FMT in clinical practice is recommended for both severe and mild PC treatment.

According to the meta-analysis data, in terms of the FMT outcomes during PC treatment, there is no substantial difference between the selected patient and anonymous healthy donors. ${ }^{7}$ The involvement of both related and unrelated FMT donors provided the excellent PC outcomes in several RCTs and large case-control series. ${ }^{3,4}$ Nonetheless, randomized trials comparing these donor categories directly are not existing for the time being. The involvement of unrelated donors may be useful in the FMT centers dealing with a large number of patients to satisfy the demands in current treatment type. The stool bank accessibility in these centers is of top priority.

In clinical practice the feces are administered in different ways by means of gastroscopy, enema, or through the upper gastrointestinal tract (the operating channel of a gastroscope, nasogastric or nasojejunal tube). Various systematic reviews and meta-analyses suggested colonoscopy as the most efficient and safest technique of fecal transplant administration..$^{3,5,11}$ Depending on the recipient's overall health, $200-500 \mathrm{mg}$ of donor fecal suspension, prepared from $20-100 \mathrm{~g}$ of feces, can be safely administered through the operating channel of a colonoscope both into right (preferably) and left colon. In case of using a colonoscope as an administration tool, recipients are pretreated with polyethyleneglycol as always prior to colonoscopy. ${ }^{1,11}$

FMT can be performed using an anema. In such case patients should keep the inserted material for at least 30 minutes and lie on the back in order to minimize the risk of defecation. The fecal suspension also can be administered via the operating channel of a gastroscope, nasogastric, nasojejunal or gastrostomy tube. The patients should stay in a vertical position during 4 hours after the infusion to prevent aspiration.

FMT tends to be a safe intervention even in immunocompromised and critical patients regardless of the administration way. In severe conditions the enema should be preferable. The incidence of severe adverse effects in recipients exposed to fecal transplant administration through the upper gastrointestinal tract is slightly higher. Thus the progression of fever and aspiration pneumonia due to nausea and vomiting was reported in some patients. ${ }^{10}$ In Moayyedi et al. trial rectal abscesses were diagnosed in 15 patients following the administration using enema. ${ }^{8}$

FMT can be repeated when required, particularly in case of the treatment failure or pseudomembranous colitis relapse..$^{1,4,5,11}$ The FMT success can depend on the amount of administered suspension as well, and can be enhanced by the multiple repeated infusions. ${ }^{11}$ The factors that negatively affect the FMT outcomes may comprise insufficient amount of donor feces, severe colitis and/or continuing antibiotic therapy. In the case of insufficient clinical response or its failure, it is recommended to utilize fecal material from another donor. It is considered that in addition to the repeated FMT performing in IBD, the success rate increases, although the convincing evidences are not provided. ${ }^{1}$

In patient's satisfactory condition FMT is commonly performed in outpatient settings. The follow-up period duration after FMT is not yet determined, as it depends on the way of administration, comorbidity and overall health. The most common short-term adverse effects following FMT in pseudomembranous colitis were diarrhea, abdominal spasms, belching, constipation, fever, as well as gram-negative bacteremia and perforation. ${ }^{1,10}$ Diarrhea, abdominal bloating and spasms, fever and disease progression were frequently reported in IBD. One patient died after regurgitation of fecal material administered into the duodenum under general anesthesia. Another patient developed septic shock and toxic megacolon following the donor stool administration throughout the pre-inserted nasogastric tube. One patient developed septic shock due to the aspiration of feces following FMT performed within a gastroscopy. ${ }^{10}$

The periodicity and duration of monitoring of long-term adverse effects after FMT are not yet defined, as it requires additional clinical and analytical findings. Currently the reports are available on the single cases of a weight gain, development of peripheral neuropathy, Sjögren syndrome, idiopathic thrombocytopenic purpura and rheumatoid arthritis. ${ }^{1,10}$ In the same time there is a great number of reports on the improvement of the other gastrointestinal diseases after FMT, in particular IBS, IBD, chronic constipation, antibiotic-associated diarrhea (AAD). ${ }^{1,9,14}$ According to the systematic review with metaanalysis (Costello S. P. et al., 2017) in particular, FMT contributed to remission induction of UC in $24-32 \%$ of patients comparing to $5-20 \%$ treated conventionally, and clinical remission was reported in $39-55 \%$ of patients comparing to $20-24 \%$ treated conventionally. The great amount of data regarding the FMT efficiency in extraintestinal diseases such as Parkinson's disease, multiple sclerosis, idiopathic thrombocytopenic purpura, metabolic disorders (obesity, diabetes mellitus) and childhood diseases are available. ${ }^{1}$

According to the guidelines patients exposed to FMT should stay under the care for at least 8 weeks. The treatment response implies 
the clinical improvement such as the lesser defecation frequency and better stool consistency, improvement of other parameters of the disease severity (laboratory, radiological or endoscopic findings). The additional test for $C$. Difficile usually is not recommended, as the toxins can circulate for the extra period of several weeks. ${ }^{1}$

As with other instrumental medical procedures, prior to FMT performing a definite period is required for special education. Therefore the physicians willing to implement FMT in their centers should receive the introductory training usually comprising several parts: clinical training including the donor and recipient selection, microbiological training (fresh and frozen fecal material preparation), performing the procedure itself using different administration ways (enema, colonoscopy, nasoduodenal/nasojejunal tube), patient monitoring following FMT.

Thus, based on the European Consensus and first-hand experience we can conclude that fecal transplantation appears to be a valuable and efficient therapeutic intervention intended to treat Clostridium difficile infection and associated pseudomembranous colitis. Furthermore the latest findings reveal FMT as a perspective and efficient therapeutic method in other disorders associated with microbiome affecting, in particular inflammatory bowel diseases, IBS, antibiotic-associated diarrhea. The FMT efficiency in extraintestinal diseases, demonstrated within the trial and certain clinical follow-up studies, requires a subsequent analysis. For the widespread FMT implementation into clinical practice in Ukraine, the construction of specialized FMT centers and appropriate professional training are required complying with the guidelines based on the reliable data.

\section{Conclusions}

Taking into account the own experience and recommendations of the European consensus on FMT, it can be concluded that FMT is an important and highly effective therapeutic intervention in the treatment of Clostridium difficile and associated PMC. In addition, FMT is a promising and effective treatment for other disorders associated with changes in intestinal microbiota, in particular inflammatory bowel disease, IBD-D, and AAD.

\section{Acknowledgments}

None.

\section{Conflicts of interest}

Author declares there are no conflicts of interest.

\section{References}

1. Cammarota G, Ianiro G, Tilg H, et al. European consensus conference on faecal microbiota transplantation in clinical practice. Gut. 2017;66(4):569-580.

2. Drekonja D, Reich J, Gezahegn S, et al. Fecal microbiota transplantation for clostridium difficile infection: a systematic review. Ann Intern Med. 2015;162(9):630-638.
3. Trubiano JA, Cheng AC, Korman TM, et al. Australasian society of infectious Diseases updated guidelines for the management of clostridium difficile infection in Adults and children in australia and new zealand. Intern Med J. 2016;46(4):479-493.

4. Debast SB, Bauer MP, Kuijper EJ, et al. European society of clinical microbiology and infectious Diseases: update of the treatment guidance document for clostridium difficile infection. Clin Microbiol Infect. 2014;20(suppl 2):1-26.

5. Kelly CR, Khorutz A, Staley C, et al. Effect of fecal microbiota transplantation on Recurrence in multiply recurrent clostridium difficile infection. Ann Intern Med. 2016;165(9):609-616.

6. Kump PK, Krause R, Steininger C, et al. Recommendations for the use of faecal Microbiota transplantation "stool transplantation": consensus of the Austrian Society of gastroenterology and hepatology (ÖGGH) in cooperation with the Austrian society of infectious diseases and tropical medicine. Z Gastroenterol. 2014;52(12):1485-1492.

7. Kassam Z, Lee Ch, Yuan Y, et al. Fecal microbiota transplantation for clostridium Difficile infection: systematic review and meta-analysis. $\mathrm{Am}$ J Gastroenterol. 2013;108(4):500-508.

8. Moayyedi P, Surette Mg, Kim Pt, et al. Fecal microbiota transplantation induces Remission in patients with active ulcerative colitis in a randomized controlled trial. Gastroenterology. 2015;149(1):102-109.

9. Rossen NG, Fuentes S, Van Der Spek MJ, et al. Findings from a randomized Controlled trial of fecal transplantation for patients with ulcerative colitis. Gastroenterology. 2015;149(1):110-118.

10. Baxter M, Colville A. Adverse events in faecal microbiota transplant: a review of the literature. $J$ Hosp Infect. 2016;92:117-127.

11. Cammarota G, Masucci L, Ianiro G, et al. Randomised clinical trial: faecal microbiota transplantation by colonoscopy vs. Vancomycin for the treatment of recurrent clostridium difficile infection. Aliment Pharmacol Ther. 2015;41(9):835-843.

12. Van Nood E, Vrieze A, Nieuwdorp M, et al. Duodenal infusion of donor feces for Recurrent clostridium difficile. NEngl J Med. 2013;368(22):407415.

13. Cui B, Feng Q, Wang H, et al. Fecal microbiota transplantation through mid-gut for refractory crohn's disease: safety, feasibility, and efficacy trial results. J Gastroenterol Hepatol. 2015;30(1):51-58.

14. Satokari R, Fuentes S, Mattila E, et al. Fecal transplantation treatment of Antibiotic-induced, noninfectious colitis and long-term microbiota follow-up. Case Rep Med. 2014;2014:913867.

15. Satokari R, Mattila E, Kainulainen V, et al. Simple faecal preparation and efficacy of

16. Frozen inoculum in faecal microbiota transplantation for recurrent clostridium difficile infection - an observational cohort study. Aliment Pharmacol Ther. 2015;41(1):46-53. 